\title{
Análise da variação termo-higrométrica entre a área urbana e rural da região metropolitana de Cuiabá/MT
}

As atividades antrópicas associadas ao processo de urbanização das cidades brasileiras refletem diretamente na qualidade de vida da população. A remoção da cobertura natural e a impermeabilização do solo modificam os elementos climáticos que compõem a atmosfera local, favorecendo a formação de anomalias termohigrométricas na área urbana. O objetivo do artigo é analisar a variação termo-higrométrica no período quente-seco da região metropolitana de Cuiabá/MT. Foram utilizados dados relativos à temperatura e umidade relativa do ar, de duas estações micrometeorológicas fixas, localizadas nos municípios de Santo Antônio do Leverger (rural) e Cuiabá (urbana). Os resultados apontam diferenças entre médias horárias de temperatura mais significativas no período noturno, chegando a $3,42{ }^{\circ} \mathrm{C}$ e umidade em $16,19 \%$. Apesar de receberem a mesma insolação, a área rural arrefece rapidamente durante a noite devido à baixa capacidade de armazenamento térmico da vegetação, ocasionando as maiores diferenças de temperatura entre ambientes urbanos e rurais.

Palavras-chave: Temperatura e Umidade Relativa do Ar; Ilha de Calor; Cuiabá.

\section{Thermo-hygrometric series analysis between urban and rural areas of the metropolitan region of Cuiabá/MT}

\begin{abstract}
Human activities associated with intense urbanization of Brazilian cities reflect directly in the quality of life of the population. The removal of the natural cover and waterproofing of the soil modify climatic elements that compose the local atmosphere, favoring the formation of thermo-hygrometric anomalies in the urban area. The objective of this article is to analyze the thermo-hygrometric variation in the hot-dry period of Cuiabá/MT metropolitan region. Data on the temperature and relative humidity of two fixed micrometeorological stations were used, located in the municipalities of Santo Antônio do Leverger (rural) and Cuiabá (urban). The results show differences between the hourly mean temperature at night, reaching $3.42^{\circ} \mathrm{C}$ and humidity at $16.19 \%$. Although they receive the same sunshine, the rural area cools quickly during the night due to the vegetation low thermal storage capacity, causing the greatest temperature differences between urban and rural environments.
\end{abstract}

Keywords: Temperature and Relative Humidity; Heat Island; Cuiabá

Topic: Meteorologia, Climatologia e Mudanças Climáticas

Reviewed anonymously in the process of blind peer.

Juliana Queiroz Borges de Magalhães Chegury

Universidade Federal de Mato Grosso, Brasil

http://lattes.cnpq.br/3221019078205134

http://orcid.org/0000-0003-1472-4280

julianaqbchegury@gmail.com

Marta Cristina de Jesus Albuquerque Nogueira (iD

Universidade Federal de Mato Grosso, Brasil

http://lattes.cnpq.br/8280601583280522

http://orcid.org/0000-0002-9227-2999

mcjanj@gmail.com

Flávia Regina Pereira Santos de Siqueira (it Universidade Federal de Mato Grosso, Brasil http://lattes.cnpq.br/1587709180081144

http://orcid.org/0000-0002-8309-3948

frpsantos@hotmail.com

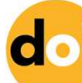

DOI: 10.6008/CBPC2179-6858.2018.003.0010
Received: 10/02/2018

Approved: 24/03/2018

\author{
Marcos de Oliveira Valin Júnior \\ Instituto Federal de Mato Grosso, Brasil \\ http://lattes.cnpq.br/1988817143994600 \\ http://orcid.org/0000-0003-2961-2011 \\ marcos.valin@cba.ifmt.edu.br \\ Karyn Ferreira Antunes Ribeiro \\ Instituto Federal de Mato Grosso, Brasil \\ http://lattes.cnpq.br/1028794796688155 \\ http://orcid.org/0000-0002-8789-1306 \\ karyn.ribeiro@cba.ifmt.edu.br \\ Flávia Maria de Moura Santos \\ Universidade Federal de Mato Grosso, Brasi \\ http://lattes.cnpq.br/4000857814123856 \\ http://orcid.org/0000-0002-6009-6185 \\ flavia mms@hotmail.com
}

Referencing this:

CHEGURY, J. Q. B. M.; NOGUEIRA, M. C. J. A.; SIQUEIRA, F. R. P. S.; VALIN JÚNIOR, M. O.; RIBEIRO, K. F. A.; SANTOS, F. M. M.. Análise da variação termo-higrométrica entre a área urbana e rural da região metropolitana de Cuiabá/MT. Revista Ibero Americana de Ciências Ambientais, v.9, n.3, p.113-120, 2018. DOI:

http://doi.org/10.6008/CBPC2179-6858.2018.003.0010 


\section{INTRODUÇÃO}

O crescimento acelerado e muitas vezes desordenado das áreas urbanas verificado nas últimas décadas tem gerado impactos negativos na qualidade de vida da população citadina, relacionados à degradação do meio ambiente, emissão de poluentes pelos veículos e indústrias, trazendo como consequência a modificação dos elementos climáticos que compõem a atmosfera local, favorecendo a formação de anomalias termo-higrométricas na área urbana.

As cidades brasileiras possuem morfologias distintas e complexas, principalmente no ponto de vista dos estudos bioclimáticos. Entre as principais consequências da expansão urbana destaca-se a modificação da paisagem natural que provoca alterações nas propriedades aerodinâmicas, radiativas e hidráulicas da superfície, nas propriedades térmicas do substrato e nas propriedades hidráulicas do solo. Além disso, as emissões de calor e poluentes derivadas das atividades humanas associadas às alterações nos padrões de evaporação da superfície modificam as propriedades térmicas e higrométricas da atmosfera urbana (OKE, 1982).

Percebe-se que os impactos combinados da urbanização, das mudanças climáticas e da ausência de um planejamento integrado por parte dos governos municipais, além de trazer danos irreversíveis ao meio ambiente, terão grandes impactos nas temperaturas das cidades no futuro e agravarão o estresse térmico existente, particularmente em países de baixa renda com pouca capacidade de adaptação ao aumento das temperaturas (ALTHOR et al., 2016; CHAPMAN et al., 2017).

As anomalias termo-higrométricas são as alterações que ocorrem na umidade e na temperatura e são oriundas do aquecimento diferenciado das diferentes características do ambiente urbano. A diferença de temperatura do ar verificada entre as áreas urbanas e rurais caracterizam a intensidade das chamadas ilhas de calor urbanas, que impactam diretamente as condições térmicas das cidades.

Segundo Voogt (2004), a intensidade da ilha de calor pode ser verificada porque durante o dia a radiação solar aquece as superfícies urbanas e este calor é armazenado nos materiais, sendo liberado no período noturno. Por isso, percebem-se altas temperaturas no período noturno, mesmo sem a presença da radiação solar.

A região metropolitana de Cuiabá, também denominada como região metropolitana do Vale do Rio Cuiabá, foi instituída em 2009, pela Lei Complementar Estadual № 359 e é composta pelos municípios de Cuiabá, Várzea Grande, Nossa Senhora do Livramento e Santo Antônio de Leverger. Embora sejam municípios vizinhos e não apresentarem diferenças significativas de altitude, com relação à temperatura e umidade relativa do ar, algumas distinções são verificadas entre eles.

Nas últimas décadas, a cidade de Cuiabá apresentou um crescimento urbano acentuado, caracterizado pela verticalização das edificações, impermeabilização do solo, aumento da frota veículos automotores e redução das áreas verdes. Segundo Maitelli (1994) o clima de Cuiabá é definido pelas temperaturas elevadas, chuva no verão e seca no inverno (tipo AW de Koppen), isto é, tropical semiúmido, com quatro a cinco meses secos e duas estações bem definidas, uma seca (outono-inverno) e uma chuvosa 
(primavera-verão). Está a uma altitude de 165 metros acima do nível do mar, variando em sua área urbana de 146 a 250 metros e o município possui uma área de $3.224,68 \mathrm{Km}^{2}$, sendo $251,94 \mathrm{Km}^{2}$ ocupados pela área urbana.

O município vizinho de Santo Antônio do Leverger possui uma considerável extensão territorial $\left(12.261 \mathrm{Km}^{2}\right)$. Suas condições climáticas são muito próximas das verificadas em Cuiabá, apresentando um clima predominantemente quente e altitude de 141 metros. No entanto, o clima quente se apresenta se forma mais intensa em Cuiabá devido ao processo de urbanização que se acentuou nas últimas décadas.

Estudos inerentes aos efeitos da ilha de calor nas áreas urbanas, à relação existente entre a densidade de ocupação, ausência de áreas verdes e formas de mitigação foram desenvolvidos por pesquisadores de diversos países. Trabalhos recentes enfatizam a constante preocupação dos autores com o aquecimento e desconforto causado pelo aumento da temperatura nas áreas urbanas, principalmente no período noturno. Chapman et al. (2017), Hu et al. (2017), Koomen et al. (2017), Richter (2016), Du et al. (2016) e Hua et al. (2016) ressaltam que o efeito negativo da ilha de calor em áreas urbanas é uma das questões ambientais mais críticas para as cidades contemporâneas de maior porte e que estudos anteriores mostram que existe uma forte correlação entre a ilha de calor e o fator de visão do céu, que demonstra uma ligação potencial entre a morfologia urbana e as ilhas de calor.

O presente estudo tem por objetivo analisar e comparar os dados de temperatura e umidade relativa do ar de dois municípios da Baixada Cuiabana: Cuiabá (Urbano) e Santo Antônio do Leverger (Rural). Os dados foram extraídos de duas estações fixas nos meses de julho, agosto e setembro de 2015, período caracterizado como quente-seco, no qual se verifica elevadas temperaturas do ar e baixa umidade, causando desconforto térmico, favorecendo o aparecimento de doenças respiratórias nos habitantes das cidades em estudo, assim como o aumento dos índices de queimadas na região.

\section{MATERIAIS E MÉTODOS}

\section{Área de estudo}

Para realização do presente trabalho foram coletados dados das variáveis termo-higrométricas de duas estações fixas. Uma estação localizada no Bairro Coxipó, no município de Cuiabá/MT (urbana) e outra localizada junto com a Estação Agrometeorológica Padre Ricardo Remetter, localizada na Fazenda Experimental da Universidade Federal de Mato Grosso (UFMT), no município de Santo Antônio de Leverger/MT (Rural), distantes a 26,62 Km entre si (Figura 1).

A estação micrometeorológica fixa localizada na porção 'rural' (Figura 1: C-1) mostra uma grande diferença da 'urbana', a área do entorno é coberta por gramíneas e algumas árvores de grande porte esparsas. A estação 'urbana' (Figura 1: C-2) se mostra em um ambiente com diversos tipos de revestimentos antrópicos com asfalto muito próximo ao local de instalação da torre. As características principais das duas estações podem ser verificadas na Tabela 1. 

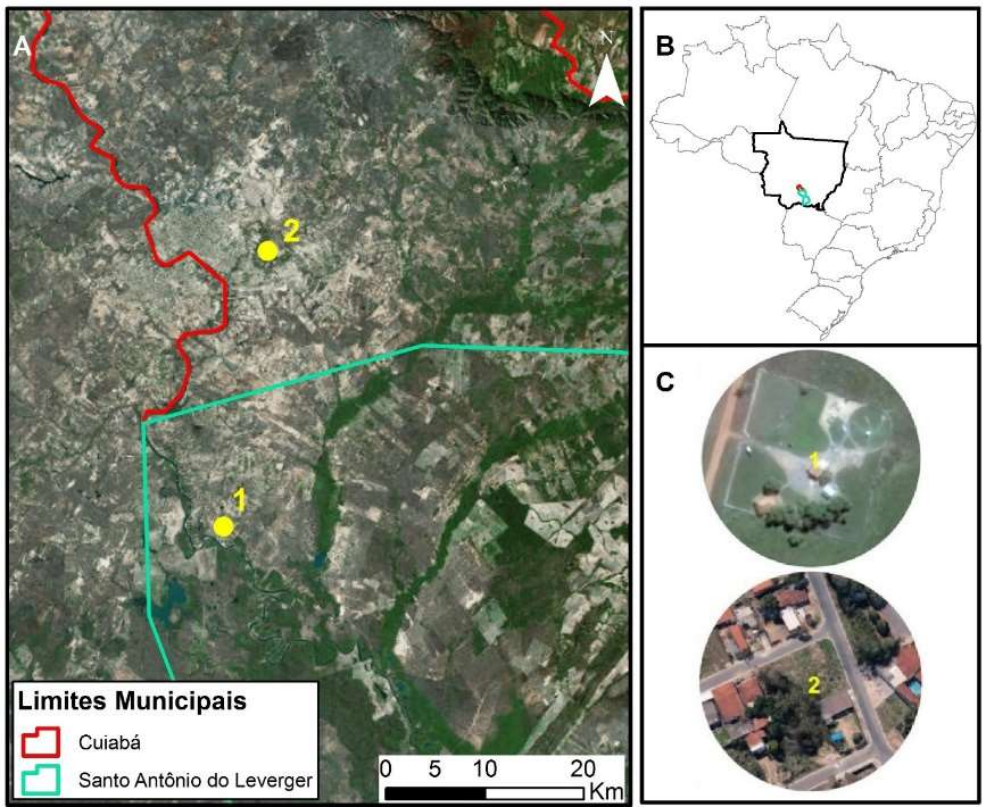

Figura 1: (A) Localização das estações fixas e limites municipais; (B) área de estudo no estado de Mato Grosso, Brasil; (C) vista do entorno das estações micrometeorológicas, rural (1) e urbana (2). Fonte: Imagens adaptadas do software Google Earth.

Tabela 1: Características das estações fixas, urbana e rural.

\begin{tabular}{|l|l|l|}
\hline Local da estação: & Urbana & Rural \\
\hline Latitude & $15^{\circ} 37^{\prime} \mathrm{S}$ & $15^{\circ} 51^{\prime} \mathrm{S}$ \\
\hline Longitude & $56^{\circ} 02^{\prime}$ O & $56^{\circ} 04^{\prime} \mathrm{O}$ \\
\hline Altitude & 169 metros & 150 metros \\
\hline $\begin{array}{l}\text { Classificação de } \\
\text { Köppen }\end{array}$ & Aw Aw \\
\hline $\begin{array}{l}\text { Características do } \\
\text { entorno }\end{array}$ & $\begin{array}{l}\text { Área densamente urbanizada, com solo impermeabilizado e Área pouco urbanizada, com } \\
\text { predomínio de materiais como concreto, asfalto e vidro. } \\
\text { vegetação e pastagens. }\end{array}$ \\
\hline
\end{tabular}

No que se referem às medições das variáveis microclimáticas (temperatura do ar e umidade relativa), estas foram realizadas no período de 26 de junho a 04 de setembro de 2015, com intervalo de 10 minutos entre as medições. A estação do ano nesse período é o inverno, que se estende do solstício de junho (dia 21) ao equinócio de setembro (dia 23), cujas características principais são dias mais curtos e noites mais longas, baixa umidade relativa do ar e aumento dos índices de queimadas verificadas na região metropolitana de Cuiabá.

Para realização das medições na estação 'urbana', foi utilizada uma estação fixa da marca Onset, com sistema de coleta de dados (datalogger) modelo U30, sensor de temperatura e umidade do modelo SOTHBM002. Na estação 'Rural' utilizou-se uma torre vertical de aço com 3,6 m altura com sistema de coleta de dados do tipo 'datalogger'. A estação urbana foi programada para realizar os registros a cada 05 minutos e a estação rural, a cada 10 minutos; portanto, foi necessário considerar na estação urbana, apenas aos dados coletados a cada 10 minutos para que os dados coletados pudessem ser analisados e comparados nos mesmos horários. Segundo Theophilou et al. (2015), a utilização de estações meteorológicas fixas na investigação as variações termo-higrométricas em áreas urbanas proporcionam uma grande vantagem: as medições estão disponíveis por longos períodos de tempo, enquanto a exatidão das medições é geralmente muito alta. 


\section{Análise dos dados}

Neste trabalho foram utilizadas médias horárias feitas a partir dos dados coletados nas estações fixas a cada 10 minutos de temperatura e umidade relativa do ar. A análise dos dados foi realizada através da elaboração de gráficos e histogramas, a fim de se comparar os valores referentes à temperatura do $\operatorname{ar}(T)$ e umidade relativa do ar (UH) da área 'urbana' e 'rural'. Conforme mencionado anteriormente, as diferenças na temperatura entre áreas urbanas e rurais caracterizam a intensidade de uma ilha de calor urbana.

A utilização de histogramas possibilita a interpretação de dados de forma mais simples em comparação às tabelas e relatórios com grande quantidade de dados numéricos. Nos estudos inerentes as ciências ambientais, diversos autores utilizaram os histogramas de frequência para análise dos resultados de suas pesquisas. Nos estudos relacionados às variações termo-higrométricas da área urbana com relação à área rural, indicando a intensidade da ilha calor urbana, a utilização de histogramas foi verificada em trabalhos relevantes, como os publicados por Camilloni et al. (2012), Elagib (2011), Półrolniczak et al. (2015), Stewart (2011) e Theophilou et al. (2015).

\section{RESULTADOS E DISCUSSÃO}

A Gráfico 2 apresenta a variação da temperatura do ar através das médias horárias dos dados coletados nos setenta e um (71) dias analisados, correspondes ao período de 26 de junho a 04 de setembro de 2015. As diferenças entre médias horárias de temperatura do ar da área urbana com relação à área considerada rural especificamente no período das $00 \mathrm{~h}$ às $7 \mathrm{~h}$, estão entre $2,27^{\circ} \mathrm{C}$ a $2,10^{\circ}$. No período das $18 \mathrm{~h}$ às $23 \mathrm{~h}$, com valores entre $2,47^{\circ} \mathrm{C}$ a $3,42^{\circ} \mathrm{C}$. Sendo que os horários com maior diferença registrada às $00 \mathrm{~h}$ e às 19h, ou seja, as diferenças se mostraram mais significativas no período noturno.

As justificativas das maiores diferenças na temperatura do ar no período noturno se devem aos tipos de cobertura dos locais analisados. Na área 'urbana', predominam pavimentos de asfalto ou concreto, atividades humanas como a utilização de ar condicionado e veículos, além de construções em alvenaria e demais sistemas construtivos que absorvem grandes quantidades de radiação incidente durante o dia e liberam durante a noite. Na área 'rural', apesar de receber quantidades iguais de insolação, arrefecem rapidamente durante a noite devido a uma capacidade muito baixa de armazenamento térmico da vegetação, causando assim as maiores diferenças de temperatura entre ambientes urbanos e rurais.

A variação da umidade relativa do ar pode ser observada na Gráfico 3. Da mesma forma que na Gráfico 2, os dados foram coletados no período de setenta e um dias. As médias horarias apontam que as diferenças entre os valores inerentes a umidade do ar da área urbana com relação à área rural, apresentaram variações significativas tanto no período diurno quanto no período noturno. As maiores diferenças foram verificadas no período noturno. Entre $00 \mathrm{~h}$ às $7 \mathrm{~h}$ a umidade relativa teve diferença entre as duas estações variando de $10,20 \%$ a $7,57 \%$ e entre as $17 \mathrm{~h}$ às $23 \mathrm{~h}$, com valores variando entre $7,81 \%$ a $16,19 \%$, com maiores diferenças registradas às $00 \mathrm{~h}$ e às $21 \mathrm{~h}$. 


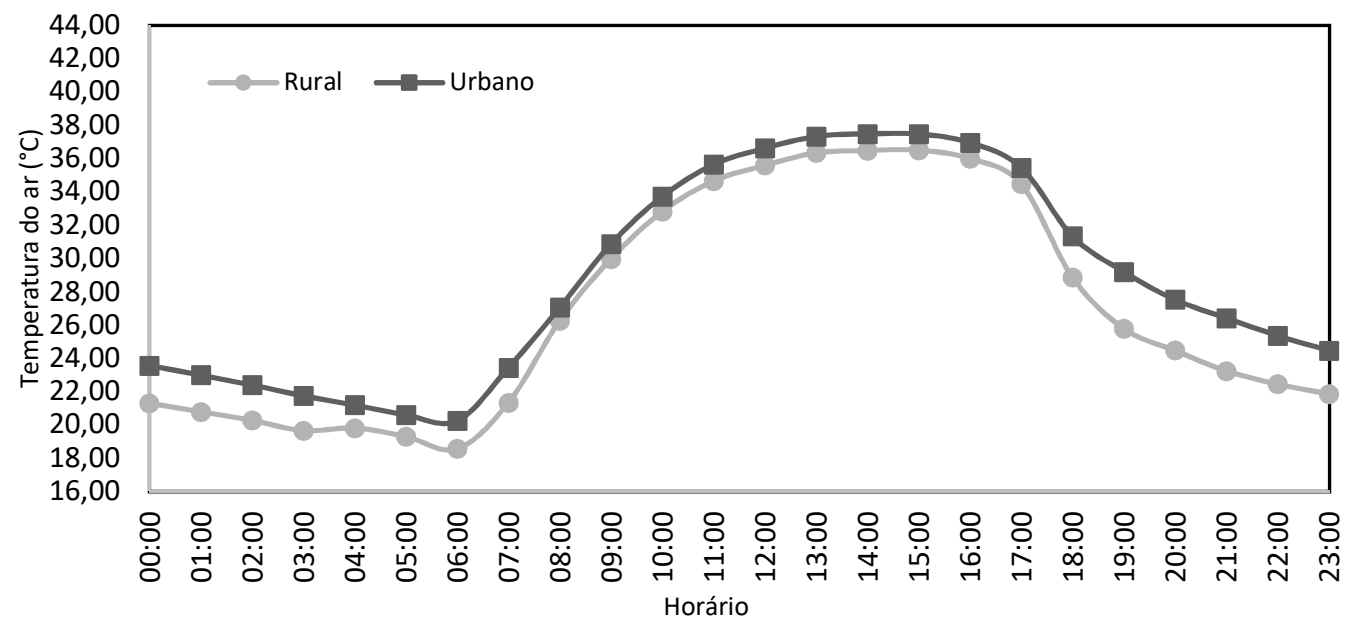

Gráfico 2: Ciclo diário da variação da temperatura do ar através das médias horárias.

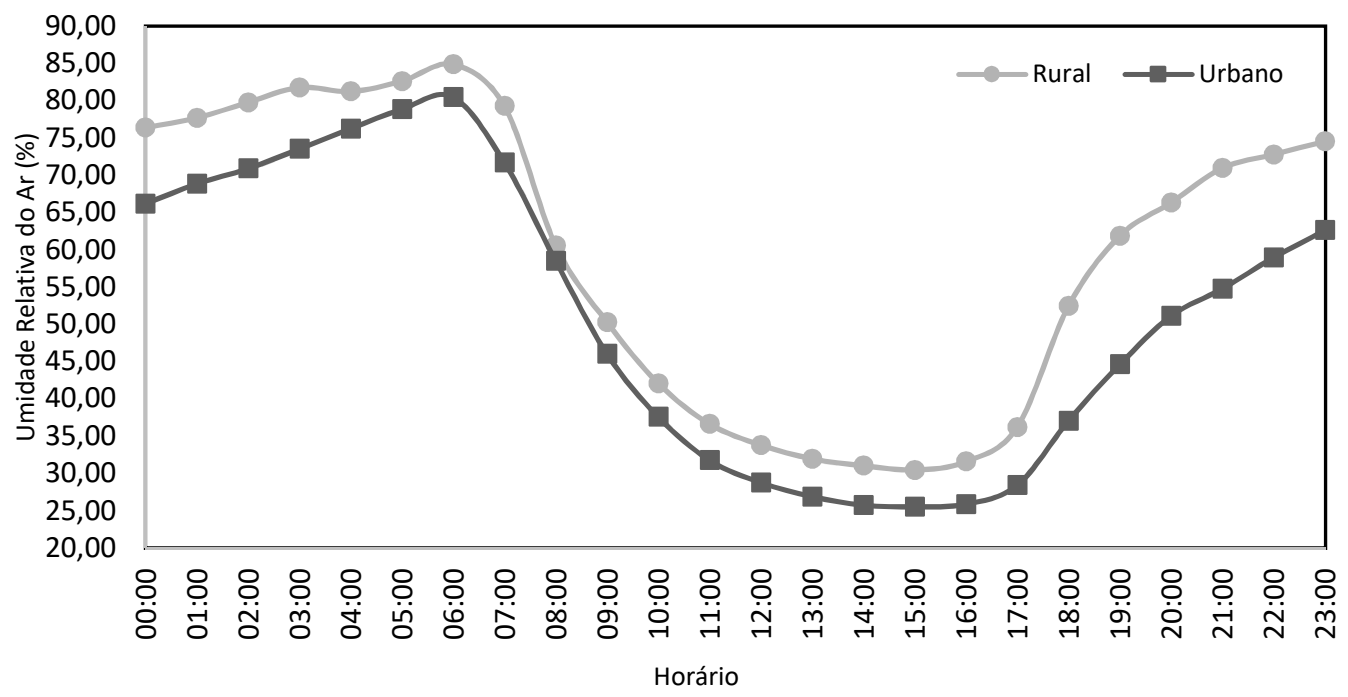

Gráfico 3: Ciclo diário da variação da umidade relativa do ar através das médias horárias.

Considerando os 71 dias de coleta de dados a cada 10 minutos, tem-se 5808 valores de temperatura e umidade relativa do ar para cada local. Distribuindo esses dados em um histograma de frequências na Temperatura do ar (Figura 2) observa-se que na estação rural a distribuição dos valores inicia-se com menos de $15^{\circ} \mathrm{C}$. Enquanto que na urbana a distribuição inicia-se após $15^{\circ} \mathrm{C}$, bem como na rural a distribuição finalizase aproximadamente com $40^{\circ} \mathrm{C}$ enquanto que na urbana se encerra próximo de $45^{\circ} \mathrm{C}$. A Tabela 2 apresenta os resultados da análise estatística dos dados coletados da área rural e da área urbana, indicando o número de dados válidos e ausentes, a média, mediana, modo, desvio padrão e variância.

Tabela 2: Estatísticas referentes aos dados de Temperatura do ar e Umidade relativa do ar.

\begin{tabular}{|l|l|l|l|l|}
\hline \multirow{2}{*}{} & \multicolumn{2}{|l|}{ Temperatura do Ar } & Umidade Relativa do Ar \\
\cline { 2 - 5 } & T_Rural & T_Urbana & UR_Rural & UR_Urbana \\
\hline N Válido & 5808 & 5808 & 5808 & 5808 \\
\hline N Ausente & 0 & 0 & 0 & 0 \\
\hline Média & 26,8945 & 28,6662 & 59,6101 & 51,4050 \\
\hline Mediana & 25,3000 & 27,7800 & 64,0000 & 52,2000 \\
\hline Modo & $20,30^{\text {a }}$ & $24,51^{\text {a }}$ & 29,00 & 24,60 \\
\hline Desvio Padrão & 7,05282 & 6,52312 & 21,88685 & 21,19734 \\
\hline Variância & 49,742 & 42,551 & 479,034 & 449,327 \\
\hline
\end{tabular}

a. Há vários modos. O menor valor é mostrado. 


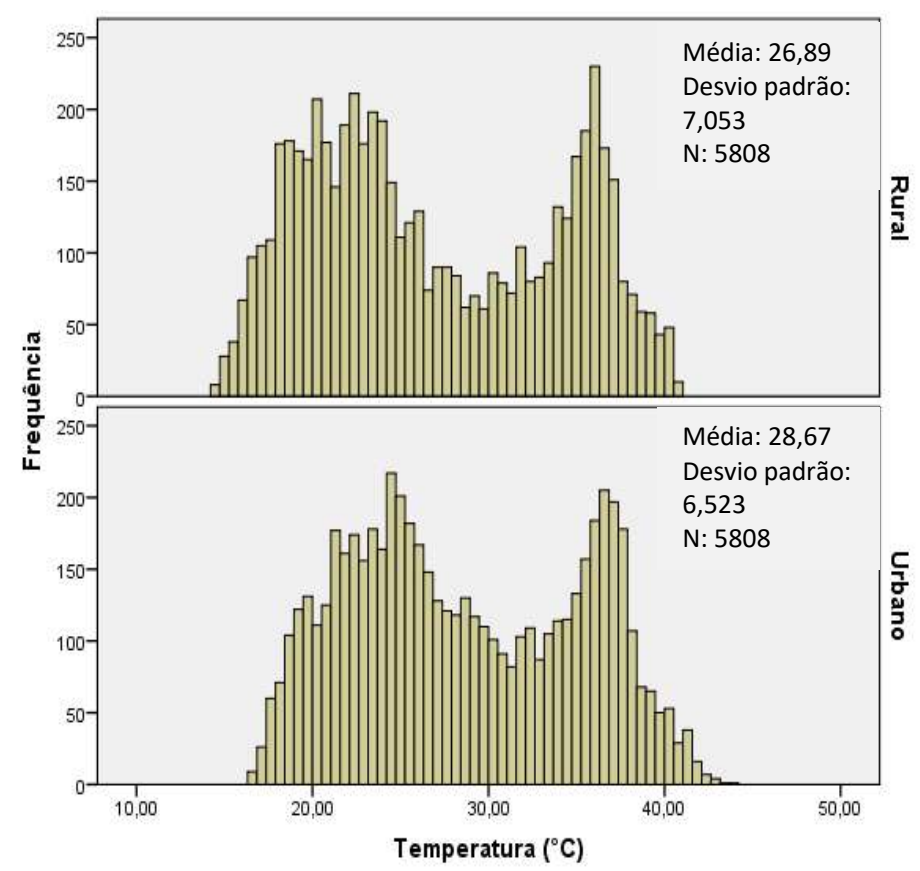

Figura 2: Histograma das diferenças de temperatura entre a área rural e urbana.

No histograma de frequências na umidade relativa do ar (Figura 3) observa-se que na estação rural a distribuição dos valores inicia-se abaixo de $20 \%$, enquanto que na urbana a distribuição inicia após $20 \%$. Também é possível observar que na área urbana as maiores frequências estão no lado esquerdo do gráfico, representando menores valores de umidade, enquanto que na área rural as maiores frequências estão do lado direito do gráfico representado os maiores valores.

Essas informações extraídas através da análise dos histogramas reforçam as teorias de que quanto maiores forem às temperaturas, menores serão os índices relativos à umidade relativa ao ar, assim, da mesma forma, percebe-se que os maiores valores de umidade do ar são verificados em dias com temperaturas mais amenas.

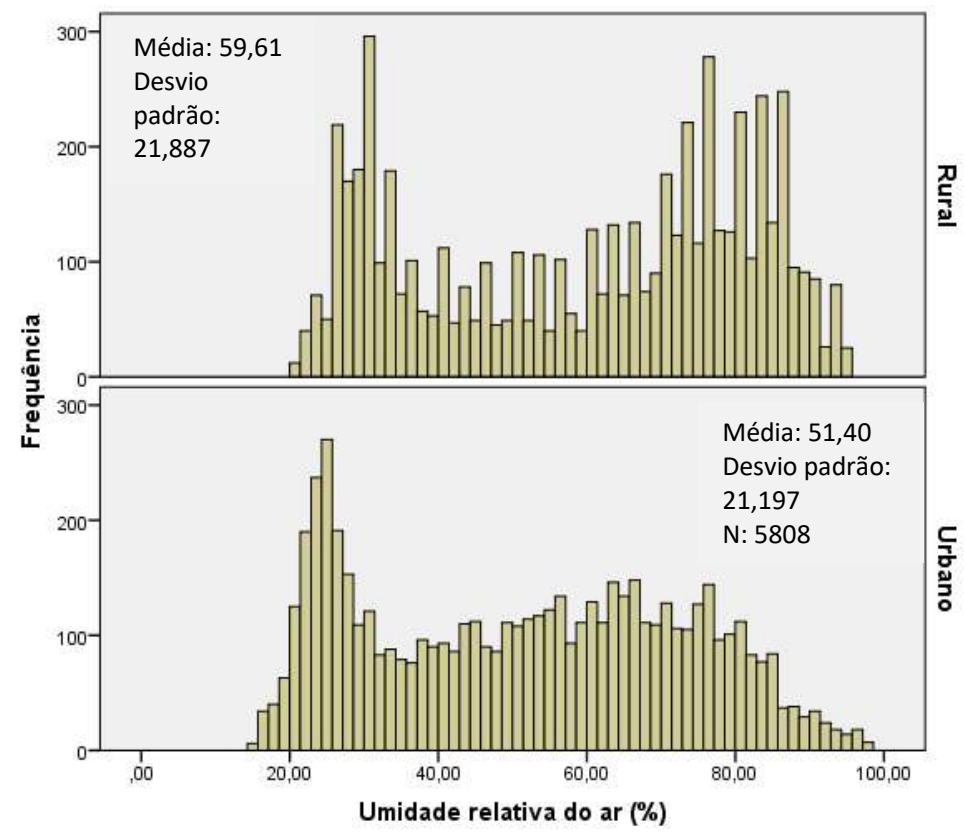

Figura 3: Histograma das diferenças de umidade relativa do ar entre a área rural e urbana. 


\section{CONCLUSÕES}

O crescimento acelerado, e por vez, desorganizado do município de Cuiabá, têm ocasionado a modificação dos elementos climáticos que compõem sua atmosfera local. Diferenças na temperatura e umidade do ar foram verificadas com relação a região rural, no município de Santo Antônio do Leverger. Os resultados apontam que as diferenças mais significativas entre médias horárias de temperatura do ar foram verificadas no período noturno, variando entre $2,10^{\circ} \mathrm{C}$ a $3,42^{\circ} \mathrm{C}$, com maiores diferenças registradas às $00 \mathrm{~h}$ e às $19 \mathrm{~h}$. Já com relação à umidade do ar, entre $7,81 \%$ a $16,19 \%$, com as maiores registradas às $00 \mathrm{~h}$ e às $21 \mathrm{~h}$.

Apesar de receberem quantidades iguais de insolação, percebe-se que a área rural perde o calor recebido durante o dia mais rapidamente durante a noite devido à baixa capacidade de armazenamento térmico da vegetação. Este fato, associado ao uso do solo, a presença de cobertura vegetal mais densa e ao processo menos intenso de urbanização, faz com que as maiores diferenças de temperatura sejam verificadas entre ambientes urbanos e rurais.

\section{REFERÊNCIAS}

ALTHOR, G.; WATSON, J. E. M.; FULLER, R. A.. Global mismatch between greenhouse gas emissions and the burden of climate change. Scientific Reports, v.6, n.20281, 2016.

CAMILLONI, I.; BARRUCAND, M.. Temporal variability of the Buenos Aires, Argentina, urban heat island. Theoretical and Applied Climatology, v.107, n.1-2, p.47-58. 2012.

CHAPMAN, S.; WATSON, J. E. M.; SALAZAR, A.; THATCHER, M.; MCALPINE, C. A.. The impact of urbanization and climate change on urban temperatures: a systematic review. Landscape Ecology, v.32, n.10, p.1921-1935, 2017.

DU, H.; WANG, D.; WANG, Y.; ZHAO, X.; QIN, F.; JIANG, H.; CAI, Y.. Influences of land cover types, meteorological conditions, anthropogenic heat and urban area on surface urban heat island in the Yangtze River Delta Urban Agglomeration. Science of the Total Environment., v.15, n.571, p.461-470, 2017. DOI: http://10.1016/j.scitotenv.2016.07.012

ELAGIB, N. A.. Evolution of urban heat island in Khartoum. International Journal of Climatology, v.31, n.9, p.13771388. 2011. DOI: https://doi.org/10.1002/joc.2159

HU, X.; ZHOU, W.; QIAN, Y.; YU, W.. Urban expansion and local land-cover change both significantly contribute to urban warming, but their relative importance changes over time. Landscape Ecology, v.32, n.4, p.763-780, 2017.

HUA, Y.; WHITEB, M.; DINGA, W.. An Urban Form Experiment on Urban Heat Island Effect in High Density Area. Procedia Engineering, v.169, p.166-174, 2016.

KOOMEN, E.; DIOGO, V. Assessing potential future urban heat island patterns following climate scenarios, socio- economic developments and spatial planning strategies. Mitigation and Adaptation Strategies for Global Change, v.22, p.287-306, 2017.

MAITELLI, G. T.. Uma Abordagem Tridimensional de Clima Urbano em Área Tropical Continental: O Exemplo de Cuiabá/MT. Tese (Doutorado em Filosofia e Ciências Humanas) - Universidade de São Paulo, São Paulo, 1994.

OKE, T. R.. The Energetic Basis of the Urban Heat Island. Quarterly Journal of the Royal Meteorological Society, v.108, n.455, p.1-24, 1982.

PÓŁROLNICZAK, M.; KOLENDOWICZ, L.; MAJKOWSKA, A.; CZERNECKI, B.. The influence of atmospheric circulation on the intensity of urban heat island and urban cold island in Poznań, Poland. Theoretical and Applied Climatology, v.127, n.3-4, p.611-625, 2017.

RICHTER, M.. Urban climate change-related effects on extreme heat events in Rostock, Germany. Urban Ecosystems, v.19, n.2, p.849-866, 2016.

STEWART, I. D.. A systematic review and scientific critique of methodology in modern urban heat island literature. International Journal of Climatology, v. 31, p. 200-217, 2011.

THEOPHILOU, M. K.; SERGHIDES, D.. Estimating the characteristics of the Urban Heat Island Effect in Nicosia, Cyprus, using multiyear urban and rural climatic data and analysis. Energy and Buildings, v.108, p.137-144, 2015. DOI: https://10.1016/j.enbuild.2015.08.034

VOOGT, J. A.. Urban heart islands: hotter cities. 2004.

A CBPC - Companhia Brasileira de Produção Científica (CNPJ: 11.221.422/0001-03) detém os direitos materiais desta publicação. Os direitos referem-se à publicação do trabalho em qualquer parte do mundo, incluindo os direitos às renovações, expansões e disseminações da contribuição, bem como outros direitos subsidiários. Todos os trabalhos publicados eletronicamente poderão posteriormente ser publicados em coletâneas impressas sob coordenação da Sustenere Publishing, da Companhia Brasileira de Produção Científica e seus parceiros autorizados. Os (as) autores (as) preservam os direitos autorais, mas não têm permissão para a publicação da contribuição em outro meio, impresso ou digital, em português ou em tradução. 